\title{
Inclusive Measurements on Diffractive Processes in ep Collisions
}

\author{
Xavier Janssen ${ }^{1}$, on behalf of the H1 and ZEUS Collaborations \\ ${ }^{1}$ Université Libre de Bruxelles, IIHE - CP 230, Boulevard du Triomphe, B-1050 Bruxelles, Belgium
}

Received on 25 November, 2006

\begin{abstract}
Measurements from the H1 and ZEUS collaborations of the diffractive deep-inelastic scattering process, $e p \rightarrow e X Y$, where $Y$ is a proton or a low mass proton excitation, are presented for photon virtualities in the range $2.2<Q^{2}<1600 \mathrm{GeV}^{2}$ and squared four-momentum transfer at the proton vertex satisfying $|t|<1 \mathrm{GeV}^{2}$. Diffractive parton distribution functions and their uncertainties are determined from a next-to-leading order DGLAP QCD analysis. Combining measurements of the inclusive diffractive deep-inelastic scattering process with an analysis of diffractive dijet production allows a very sensitive determination of both quark and gluon distributions.
\end{abstract}

Keywords: Diffraction; Deep inelastic scattering

\section{INTRODUCTION}

This proceeding summaries recent results [1-4] obtained with the H1 and ZEUS detectors at the HERA $e p$ collider on measurements of the diffractive deep-inelastic scattering (DIS) process, $e p \rightarrow e X Y$, where $Y$ is a proton or a low mass proton excitation, which represents at low Bjorken $x$ approximatively $10 \%$ of the total cross section. In diffractive interactions, a photon of virtuality $Q^{2}$ is emitted by the incoming lepton and interacts strongly with the proton to form two hadronic final state systems $X$ and $Y$ separated by a large gap

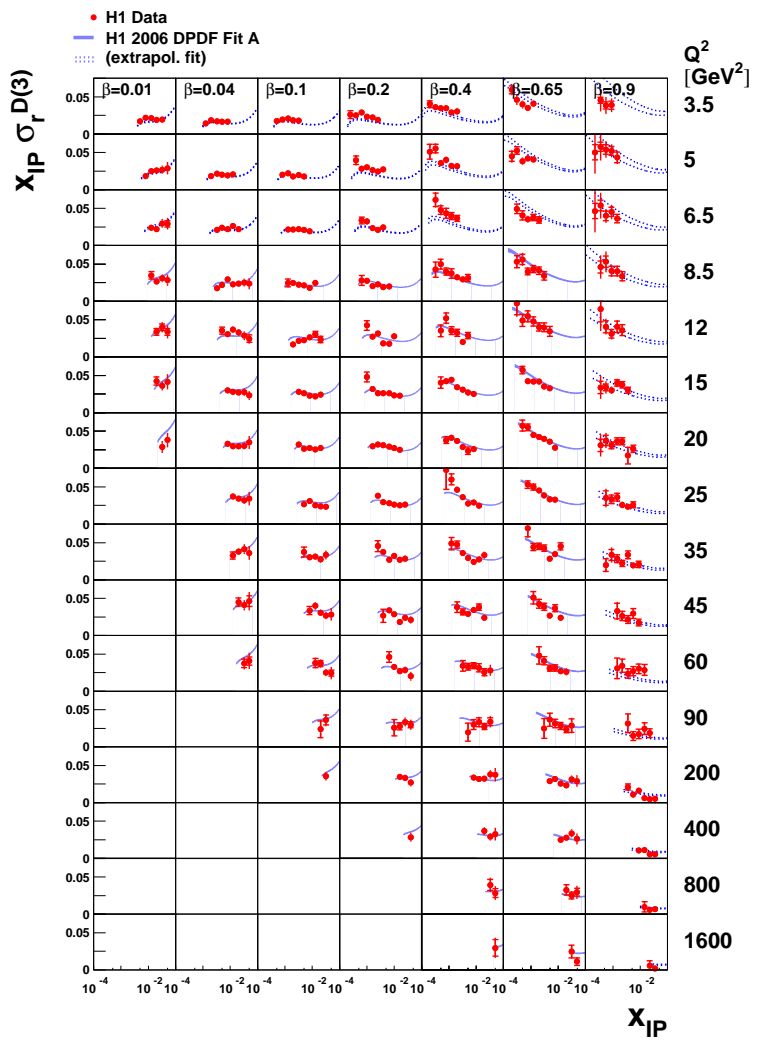

FIG. 1: The $x_{\mathbb{P}}$ dependence of the diffractive reduced cross section, multiplied by $x_{\mathbb{P}}$, at fixed values of $\beta$ and $Q^{2}$ as measured by H1 [1]. The data are compared with the results of the "H1 2006 DPDF Fit A" resulting from a NLO DGLAP analysis. in rapidity. Such processes are often discussed in terms of an exchange with net vacuum quantum numbers, the pomeron $(\mathbb{P})$, and a fraction $x_{\mathbb{P}}$ of the proton longitudinal momentum is transferred to the system $X$. Understanding the nature of the pomeron in terms of quarks and gluons is a challenge for Quantum Chromodynamics (QCD).

\section{CROSS SECTION MEASUREMENTS}

High statistic samples of diffractive DIS events are selected either by requesting a large rapidity gap (LRG) in the outgoing proton direction (H1 case [1]) or by a subtraction method differentially in the mass of the $X$ system $\left(M_{X}\right.$ Method, ZEUS case [3]). In such case, the measured process by H1 (ZEUS) is $e p \rightarrow e X Y$, where $Y$ corresponds to any baryonic system with mass $M_{Y}<1.6 \mathrm{GeV}\left(M_{Y}<2.3 \mathrm{GeV}\right)$. Both experiments have also collected smaller samples for the $e p \rightarrow e X p$ process by directly detecting the outgoing proton with Roman Pot detectors $[2,4]$. This last selection method allows to measure the outgoing proton four-momentum and to reconstruct the squared four-momentum transfer at the proton vertex $t$. In addition to $t, x_{\mathbb{P}}$ and the usual DIS variables $x$ and $Q^{2}$, measurements are made as a function of $\beta=x / x_{\mathbb{P}}$, which corresponds to the fraction of the exchanged longitudinal momentum carried by the quark coupling to the virtual photon. Alltogether, the collected data are covering photon virtualities in the range $2.2<Q^{2}<1600 \mathrm{GeV}^{2}, x_{\mathbb{P}}<0.05$ and $|t|<1 \mathrm{GeV}^{2}$.

\section{A. Reduced cross section $\sigma_{r}^{D(3)}$}

As the $t$ dependence can not be measured in the case of the LRG and $M_{X}$ Methods, the data are presented in the form of a "diffractive reduced cross section" $\sigma_{r}^{D(3)}$, integrated over $t$ and $M_{Y}$ in the ranges specified above and related to the differential cross section measured experimentally by

$$
\frac{\mathrm{d}^{3} \sigma^{\mathrm{ep} \rightarrow \mathrm{eXY}}}{\mathrm{dx}_{\mathbf{P}} \mathrm{d} \mathrm{dxd} \mathrm{Q}^{2}}=\frac{2 \pi \alpha^{2}}{x Q^{2}} Y_{+} \sigma_{r}^{D(3)}\left(x_{\mathbb{P}}, x, Q^{2}\right)
$$

where $Y_{+}=1+(1-y)^{2}$. Similarly to inclusive DIS, the reduce cross section is related to the diffractive structure func- 
tions $F_{2}^{D(3)}$ and $F_{L}^{D(3)}$ in the one-photon exchange approximation according to

$$
\sigma_{r}^{D(3)}=F_{2}^{D(3)}-\frac{y^{2}}{Y_{+}} F_{L}^{D(3)}
$$

The diffractive reduced cross section as measured via the LRG Method by the $\mathrm{H} 1$ experiment [1] is presented in Fig. 1 as function of $x_{\mathbb{P}}$ at fixed values of $\beta$ and $Q^{2}$, together with results of the "H1 2006 DPDF Fit A" presented below. These data presents unprecedented precision of $5 \%$ statistical, $5 \%$ systematic and $6 \%$ normalization in the best-measured region. Fig. 2 present a comparaison of the results on the diffractive reduced cross section obtained via the $M_{X}$ Method by the ZEUS experiment [3] with the H1 results. The two datasets are globally consistent with however some differences at low $Q^{2}$ and high $\beta$ being observed.

\section{B. $t$ dependence}

The datasets collected by the Roman Pot detectors allow to measure the $t$ dependence of the diffractive cross sections which is commonly parameterised with an exponential, $d \sigma / d t \sim e^{B t}$. The values of $B$ resulting from such fits to the H1 [2] and ZEUS [4] data are shown as a function of $x_{\mathbb{P}}$ in Fig. 3. At low $x_{\mathbb{P}}$, the $\mathrm{H} 1$ data are compatible with a constant slope parameter, $B \simeq 6 \mathrm{GeV}^{-2}$ wheather the high $x_{\mathbb{P}}$ region is thought to be dominated by the exchange of subleading trajectory. In a Regge approach with a single linear exchanged pomeron trajectory, $\alpha_{\mathbb{P}}(t)=\alpha_{\mathbb{P}}(0)+\alpha_{\mathbb{P}}^{\prime} t$, the slope parameter decreases with increasing $x_{\mathbb{P}}$ according to $B=B_{0}-2 \alpha_{\mathbb{P}}^{\prime} \ln x_{\mathbb{P}}$. The low $x_{\mathbb{P}} \mathrm{H} 1$ data favour a small value of $\alpha_{I P}^{\prime} \simeq 0.06 \mathrm{GeV}^{-2}$, though $\alpha_{\mathbb{P}}^{\prime} \simeq 0.25 \mathrm{GeV}^{-2}$, as obtained from soft hadronic interactions, cannot be excluded.

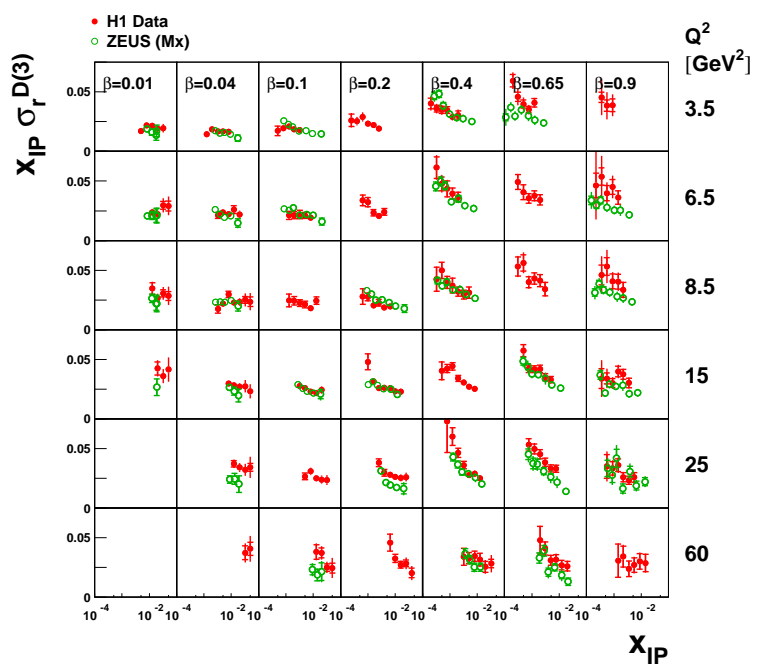

FIG. 2: Comparison of the $x_{\mathbb{P}}$ dependence of the diffractive reduced cross section, multiplied by $x_{\mathbb{P}}$, at fixed values of $\beta$ and $Q^{2}$ as measured by ZEUS [3] and H1 [1].

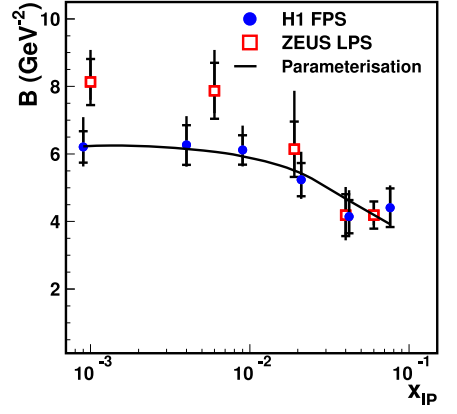

FIG. 3: Measurements of the slope parameter $B$ by $\mathrm{H} 1$ and ZEUS.

\section{QCD ANALYSIS OF H1 DATA AND DIFFRACTIVE PARTON DISTRIBUTIONS}

As proved in [5], QCD hard scattering collinear factorisation is applicable to hard diffractive processes in $e p$ interactions such that cross sections can be written in terms of convolutions of partonic cross sections $\hat{\sigma}^{e i}\left(x, Q^{2}\right)$ with Diffractive Parton Distribution Functions (DPDF) $f_{i}^{D}$ :

$$
\mathrm{d} \sigma^{e p \rightarrow e X Y}\left(x, Q^{2}, x_{\mathbb{P}}, t\right)=\sum_{i} f_{i}^{D}\left(x, Q^{2}, x_{\mathbb{P}}, t\right) \otimes \mathrm{d} \hat{\sigma}^{e i}\left(x, Q^{2}\right) .
$$

The partonic cross sections are the same as those for nondiffractive interactions. The DPDFs represent probability distributions for partons $i$ in the proton under the constraint that the proton is scattered to a specified system $Y$ with a specified four-momentum. If such a theorem holds, the DPDFs $f_{i}^{D}$ should be universal for all hard diffractive processes, e.g. inclusive diffraction, jet or heavy-quark production. They are not known from first principles, but can be determined from fits to the data using the DGLAP evolution equations.

The kinematic range in $x$ and $Q^{2}$ accessible being limited for a given value of $x_{\mathbb{P}}$, a parameterisation of the $x_{\mathbb{P}}$ dependence of the DPDFs is necessary. The proton vertex factorisation framework, in which the DPDFs are factorised into a term depending only on $x_{\mathbb{P}}$ and $t$ and a term depending only on $x$ (or $\beta$ ) and $Q^{2}$, is often assumed:

$$
f_{i}^{D}\left(x, Q^{2}, x_{\mathbb{P}}, t\right)=f_{\mathbb{P} / p}\left(x_{\mathbb{P}}, t\right) f_{i}\left(\beta=x / x_{\mathbb{P}}, Q^{2}\right) .
$$

This is equivalent to treating the diffractive exchange as a "pomeron" with a partonic structure given by the parton distributions $f_{i}\left(\beta, Q^{2}\right)$, the "pomeron flux factor" $f_{\mathbb{P} / p}\left(x_{\mathbb{P}}, t\right)$ representing the probability that a pomeron with particular values of $x_{\mathbb{P}}$ and $t$ couples to the proton. The $\mathbb{P}$ flux can be parametrised using a Regge inspired form $f_{\mathbb{P} / p}=$ $A_{\mathbb{P}} e^{B t} / x_{\mathbb{P}}^{2 \alpha_{\mathbb{P}}(t)-1}$. The H1 data $[1,2]$ are consistent with the vertex factorisation assumption and it is then used in their QCD fit.

The H1 collaboration performs next-to-leading order (NLO) QCD DGLAP fits to the reduced diffractive cross section in order to extract DPDFs. To avoid regions which are most likely to be influenced by higher twist contributions or other problems with the chosen theoretical framework, only data with $M_{x}>2 \mathrm{GeV}, \beta<0.8$ and $Q^{2}>8.5 \mathrm{GeV}^{2}$ are included in the fits. 
The DPDFs are modelled in terms of a singlet distribution $\Sigma(z)$, consisting of $u, d$ and $s$ quarks and anti-quarks with $u=d=s=\bar{u}=\bar{d}=\bar{s}$, and a gluon distribution $g(z)$. Here, $z$ is the longitudinal momentum fraction of the parton entering the hard sub-process with respect to the diffractive exchange, such that $z=\beta$ at the lowest quark-parton order in inclusive diffraction, whereas $0<\beta<z$ for higher order or other diffractive processes. The quark singlet and gluon distributions are parametrised at the starting scale $Q_{0}^{2}$ as:

$$
z f_{i}\left(z, Q_{0}^{2}\right)=A_{i} z^{B_{i}}(1-z)^{C_{i}}
$$

where $A_{i}, B_{i}$ and $C_{i}$ are free parameters of the fits, together with $\alpha_{\mathbb{P}}(0)$, which control the $x_{\mathbb{P}}$ dependence and the normalisation of the sub-leading exchange contribution needed at high $x_{\mathbb{P}}$. Information about fixed parameters and more details on the fits can be found in [1].
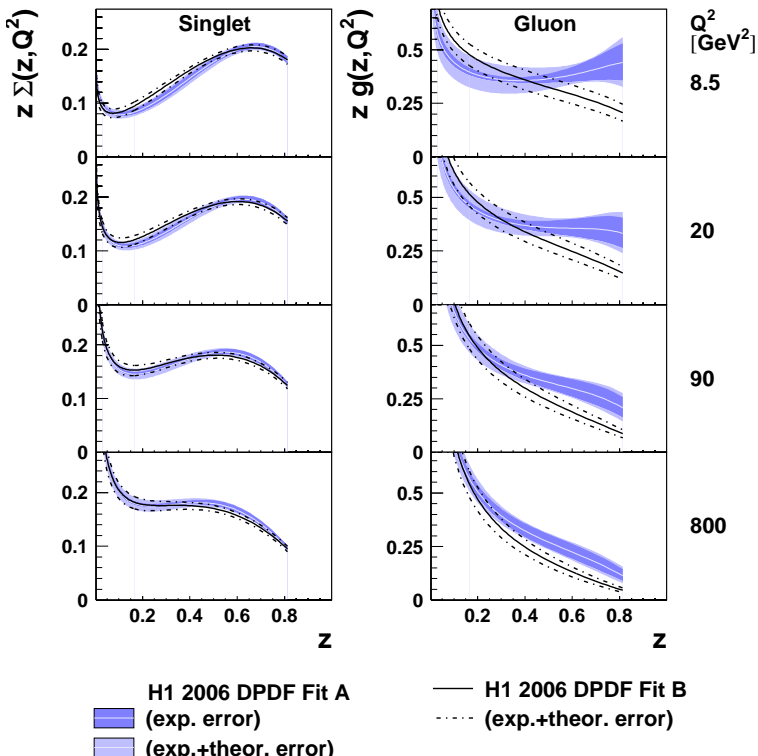

$\square$ (exp.+theor. error)

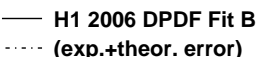

FIG. 4: The total quark singlet and gluon distributions, obtained from the "H1 2006 DPDF Fit A" and the "H1 2006 DPDF Fit B", shown at four values of $Q^{2}$ as a function of $z$.

Resulting total quark singlet and gluon distributions, obtained from NLO QCD DGLAP fits to the H1 data are shown in Fig. 4 at four values of $Q^{2}$ as a function of $z$. The singlet quark density is very closely related to the measured diffractive cross section and is thus well constrained, with a typical error of $5 \%$, the data requiring the inclusion of all three parameters $A_{q}, B_{q}$ and $C_{q}$. By comparison, the gluon density is weakly constrained by the data, especially at high $z$, and

[1] A. Aktas et al., H1 Collab., hep-ex/0606004.

[2] A. Aktas et al., H1 Collab., hep-ex/0606003.

[3] S. Chekanov et al., ZEUS Collab., Nucl. Phys B 713, 3 (2005).

[4] S. Chekanov et al., ZEUS Collab., Eur. Phys. J C 38, 43 (2004). insensitive to the $B_{g}$ parameter. In the "H1 2006 DPDF Fit A", the gluon density is parametrised at $Q_{0}^{2}=1.75 \mathrm{GeV}^{2}$ using only the $A_{g}$ and $C_{g}$ parameters, yielding a minimum $\chi^{2}$ value of 158/183 dof. However, leaving out also the $C_{g}$ parameter in the "H1 2006 DPDF Fit B", i.e. that the gluon density is a single constant $A_{g}$ at $Q_{0}^{2}=2.5 \mathrm{GeV}^{2}$, leads to only a small increase in the $\chi^{2}(164 / 183$ dof). The two fits being quite different at high $z$ points again to the weak constraint on the gluon density from the inclusive diffractive data. The fraction of the exchanged momentum carried by gluons integrated over $0.0043<z<0.8$ is around $70 \%$ throughout the $Q^{2}$ range studied, confirming the gluon dominance in the diffractive exchange. Fit A yields an effective pomeron intercept, independently of $Q^{2}$, of $\alpha_{P}(0)=1.118 \pm 0.008(\text { exp. })_{-0.010}^{+0.029}$ (model). This is slightly higher than the value $\alpha_{\mathbb{P}}(0) \simeq 1.08$ describing "soft" hadronic interactions. This result is compatible with that obtained from the ZEUS data [3] which are however indicating an increase of $\alpha_{\mathbb{P}}(0)$ for $Q^{2}>20 \mathrm{GeV}^{2}$, which would result in a breakdown of the proton vertex factorisation.

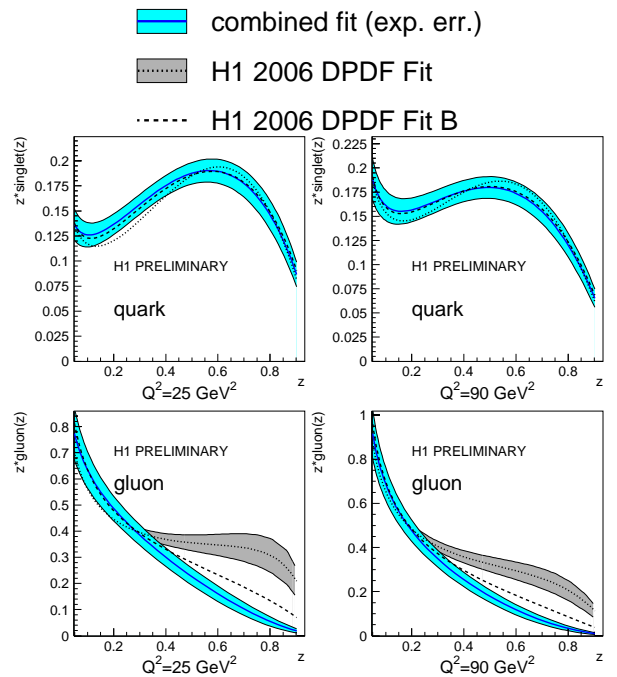

FIG. 5: The total quark singlet and gluon distributions, obtained from a simultaneous fit to inclusive and dijet diffractive data, together with results from fits to the inclusive diffractive data only, shown at two values of $Q^{2}$ as a function of $z$.

To allow for a very sensitive determination of both the quark singlet and the gluon distributions in the range $0.05<$ $z<0.9$ with comparable precision, a third fit is performed simultaneously to the $\mathrm{H} 1$ inclusive diffractive data [1] and to the diffractive dijet cross section in DIS as measured by the H1 collaboration [6]. A unique parametrisation compatible only with the "H1 2006 DPDF Fit B" is obtained, see Fig. 5.
[5] J. Collins, Phys. Rev. D 57, 3051 (1998) [Erratum Phys. Rev. D 61, 019902 (2000)]

[6] A. Aktas, et al., H1 Collab., hep-ex/0703022. 